\title{
Dynamic and acoustic characterisation of automotive wheels
}

\author{
Francesca Curà* and Graziano Curti \\ Mechanics Department of Politecnico di Torino Torino, Italy
}

\begin{abstract}
The subject of this paper is the dynamic and acoustic characterisation of an automotive wheel. In particular, an experimental research activity previously performed by the authors about the dynamic behaviour of automotive wheels has been extended to the acoustic field.

This experimental research has begun in the laboratory of the Mechanics Department of Politecnico di Torino where the modal analysis of the SAAB $640 / 6 \mathrm{Jx} 15 \mathrm{H} 2$ wheel has been carried on step by step, starting from the rim in the most simple constraint condition to realise in laboratory, that is the free-free condition, up to the wheel (rim with tire) clamped to a real automotive hub; two cases have been considered: the wheel in hanging condition, that is with the vehicle on a lift-bridge, and the wheel laying on the ground.

Then an acoustic study, analytical, numerical and experimental, of the cavity tire has also been performed. The experimental analysis has been carried on by introducing a microphone in the tire cavity (with no pressure) and by measuring the corresponding response level. The analytical study has been performed by utilising analytical relationships known in literature and a model of the tire cavity with an equivalent geometry. The numerical analysis has been carried on by means of a Boundary Element Method (BEM) model of the cavity.

All obtained results, both dynamic and acoustic, have been analysed and compared to those concerning the experimental tests of the wheel clamped to the vehicle hub.
\end{abstract}

\section{Introduction}

The knowledge of the dynamic behaviour of automotive wheels is widely important as regard both the stability (and then performance and safety) and driving comfort.

As regard the study of the vehicle stability, it is adequate to perform the dynamic analysis of the wheels in order to know the corresponding modal parameters; as regard the driving comfort, it is necessary to associate an acoustic analysis to the vibrational one in the suitable frequency range.

The dynamic study allows us to obtain both modal parameters (i.e. natural frequencies and corresponding mode shapes) of a wheel and acceleration response levels in resonance conditions.

The acoustic analysis allows us to determine both modal parameters (i.e. natural frequencies and corresponding mode shapes) of the wheel acoustic cavity and noise levels (i.e. acoustic pressure levels) coupled to acoustic and/or structural resonances.

The subject of this paper is the dynamic and acoustic characterisation of an automotive wheel, by carrying on the experimental research previously performed by the authors [1-3] in order to extend this activity to the acoustic field.

This experimental research has begun in the laboratory of the Mechanics Department of Politecnico di Torino where the dynamic characterisation of two rims, a SAAB 640/6Jx15 H2 and a VOLVO P23/6.5Jx15 H2 has been performed. The modal analysis has been carried on step by step, starting from the rim in the most simple constraint condition to realise in a laboratory, that is the free-free condition, up to the wheel (rim with tire) clamped to a real automotive hub; significant parameters have been varied each time.

\footnotetext{
${ }^{*}$ Corresponding author. E-mail: francesca.cura@polito.it.
} 
In particular in [1] the modal analysis of the two rims (SAAB 640/6Jx15 H2 and VOLVO P23/6.5Jx15 H2) both with free-free and constrained conditions has been performed (a clamping device has been realised in order to simulate the real clamp to the automotive hub).

In [1] the analysis of the two wheels (rims with tires) with free-free condition has been also carried out.

In [2] the dynamic analysis of the two wheels clamped to the test hub for varying the tire pressure and the tightening torque of the clamp bolts has been then completed. Two different tires have been considered for each rim.

In [3] the previous experimental analysis has been extended to the case of a wheel (Saab rim with Uniroyal tire) clamped to an automotive hub. The wheel has been clamped to a Saab $9^{5}$ vehicle, where the experimental tests have been performed. Two cases have been considered: the wheel in hanging condition, that is with the vehicle on a lift-bridge, and the wheel laying on the ground. The experimental results, that is the measured Frequencies Response Functions, have been compared to those obtained in the laboratory of the Mechanics Department of Politecnico di Torino on the same Saab wheel clamped to the test hub simulating the actual vehicle boundaries.

The previously described research activity led us to define a study methodology for the dynamic characterisation of automotive wheels, both from the point of view of the mode shapes (associate to their natural frequencies) classification and from the point of view of the influence parameters (the tire, the pressure of the tire, the clamping condition, ....) affecting the vibrational behaviour of the wheel.

In particular, radial mode shapes have been classified by adopting the sequence of both number of lobes (one lobe, two lobes, ...) and the order of bending modes (first mode, second mode, ... ).

Axial mode shapes are called pumping and oscillation.

From the significant parameters point of view, mode shapes have been classified by starting from the analysis of the free-free rim up to the wheel clamped on the vehicle and laying on the ground, with the aim to consider the influence of each parameter on the global behaviour of the wheel. As a matter of fact, the mode shapes of the rim, the mode shapes of the wheel (rim with tire) due to the presence of the tire, the mode shapes due to clamping device and those of the wheel as a whole have been classified and individually emphasised.

In short, the aim of the previous experimental analysis was to study the dynamic behaviour of a wheel from the rim point of view, that is by considering as a goal the possibility to predict along the design phase the vibrational phenomena of a wheel clamped on an automotive hub.

For this reason, an experimental model with a great number of measure points (114) has been utilised in all tests; all these nodes belong to the external surface of the rim.

The above quoted experimental model did not allow to identify the difference between the natural modes of the rim due to the presence of the tire and those characteristic of the tire. Therefore, these natural tire modes have not been taken into account.

Furthermore, the previous experimental research [1-3] didn't consider any acoustic phenomenon.

The problem of the noise generated by the rim-tire coupling condition has been little investigated in literature.

In [4] R. W. Scavuzzo, L. T. Charek, P. M. Sandy and G. D. Shteinhauz analysed the contribution of the rim-tire system to the vehicle noise. In particular, a critical coupling concerning the isochronism condition between an acoustic frequency of the tire cavity and a bending mode of the wheel (with respect to the hub) has been pointed out.

In [5] L. E. Kung and W. Soedel studied the free vibration of a pneumatic tire wheel using both analytical and finite element models. The analytycal model was constituted by a ring on an elastic foundation.

In [6] T. L. Richards described a finite element formulation used for numerical calculation of the natural frequencies and modes of an axisymmetric structure enclosing an acoustic cavity, simulating the structural-acoustic coupling in tyres.

A first aim of the present work is to complete the previous experimental modal analysis of automotive wheels, particularly referring to the SAAB wheel. To do that, the study of the rim (free-free, clamped to the test hub and to the vehicle hub) has been coupled to the study of the tire, in order to identify with more accuracy the dynamic behaviour of the wheel subject to real clamping condition and laying on the ground.

The modal analysis of the SAAB wheel, already performed in the laboratory of the Mechanics Department of Politecnico di Torino with impulse excitation [1] and [2], has been repeated in this work with a different experimental model in order to evaluate the tests repeatability. This new experimental model has a greater number of measure points, considering both nodes on the rim and on the surface of the tire. 
Table 1

Natural frequencies and mode shapes of the Uniroyal tire ( $p=2.5 \mathrm{~atm})$

\begin{tabular}{|c|c|c|c|}
\hline Tire frequency $[\mathrm{Hz}]$ & \multicolumn{2}{|c|}{ Wheel frequency $[\mathrm{Hz}]$} & Mode shape \\
\hline 70 & \multicolumn{2}{|c|}{70} & 1st tire mode \\
\hline 108 & \multicolumn{2}{|c|}{110} & 2nd tire mode \\
\hline 113 & \multicolumn{2}{|c|}{113} & 2nd tire mode* \\
\hline 135 & \multicolumn{2}{|c|}{136} & 3rd tire mode \\
\hline 193 & \multicolumn{2}{|c|}{193} & 4th tire mode \\
\hline 406 & \multicolumn{2}{|c|}{409} & 5th tire mode \\
\hline 436 & \multicolumn{2}{|c|}{-} & 6 th tire mode \\
\hline 484 & \multicolumn{2}{|c|}{463} & 7 th tire mode \\
\hline 667 & \multicolumn{2}{|c|}{686} & 8th tire mode \\
\hline 759 & \multicolumn{2}{|c|}{756} & 9th tire mode \\
\hline 802 & \multicolumn{2}{|c|}{810} & 10th tire mode \\
\hline 1131 & \multicolumn{2}{|c|}{1125} & 11th tire mode \\
\hline \multicolumn{4}{|c|}{$\begin{array}{l}\qquad \text { Table } 2 \\
\text { Calculated (analytical [12] and BEM solutions [13]) and measured } \\
\text { frequencies of the tire cavity }\end{array}$} \\
\hline \multirow[t]{2}{*}{ Mode shape } & \multicolumn{3}{|c|}{ Frequency [Hz] } \\
\hline & ytical [12] & BEM [13] & Measured \\
\hline 010 & 228 & 230 & 236 \\
\hline 020 & 455 & 460 & 450 \\
\hline 011 & 605 & - & - \\
\hline 030 & 683 & 690 & 686 \\
\hline $100-101$ & 876 & - & - \\
\hline 110 & 905 & - & - \\
\hline 040 & 910 & - & 915 \\
\hline 120 & 987 & - & - \\
\hline 111 & 1064 & - & - \\
\hline 130 & 1110 & - & 1125 \\
\hline 050 & 1138 & - & 1160 \\
\hline
\end{tabular}

A further aim of the present work is to couple the acoustic and the dynamic analyses, in order to evaluate the contribution of vibrations (expressed in terms of structure acceleration) to the produced noise level. To do that, an acoustic study, analytical, numerical and experimental, of the cavity tire has been performed.

The experimental analysis has been carried on by introducing a microphone in the tire cavity (with no pressure) and by measuring the corresponding response level.

The analytical study has been performed by utilising relationships known in literature [12] and a model of the tire with an equivalent geometry.

The numerical analysis has been carried on by means of a Boundary Element Method (BEM) model [13] of the cavity.

All obtained results, both dynamic and acoustic, have been analysed and compared to those concerning the experimental tests of the wheel clamped to the vehicle hub [3].

\section{Experimental analysis}

The modal analysis of both SAAB rim and SAAB wheel (Saab rim with Uniroyal tire), already performed in the laboratory of the Mechanics Department of Politecnico di Torino with impulse excitation [1-3], was repeated in this work in order to evaluate the tests repeatability.

In particular, the modal analysis of the rim both with free-free and clamped condition has been firstly performed by utilising the old experimental model (114 measure points on the rim) and the roving hammer technique [1-3].

Then the modal analysis of the wheel (rim with tire) has been repeated both free-free and clamped on the test hub (tightening torque of the bolts $R C=13 \mathrm{Nm}$, tire pressure $p=2.5 \mathrm{~atm}$ ). The new experimental model with 168 measure points (114 nodes on the rim and 54 on the surface of the tire) (see Figs 1 and 2) and the fixed hammer 
Table 3

Natural frequencies and mode shapes of the free-free Saab rim

\begin{tabular}{cc}
\hline Frequency $[\mathrm{Hz}]$ & Mode shape \\
\hline 200 & 2 lobes, 1st mode \\
560 & 3 lobes, 1st mode \\
975 & 3 lobes, 2nd mode \\
1044 & 4 lobes, 1st mode \\
1054 & 4 lobes, 1st mode* \\
1092 & pumping \\
1231 & 4 lobes, 2nd mode \\
1289 & 2 lobes, 2nd mode \\
1299 & 2 lobes, 2nd mode* \\
1324 & oscillation \\
\hline
\end{tabular}

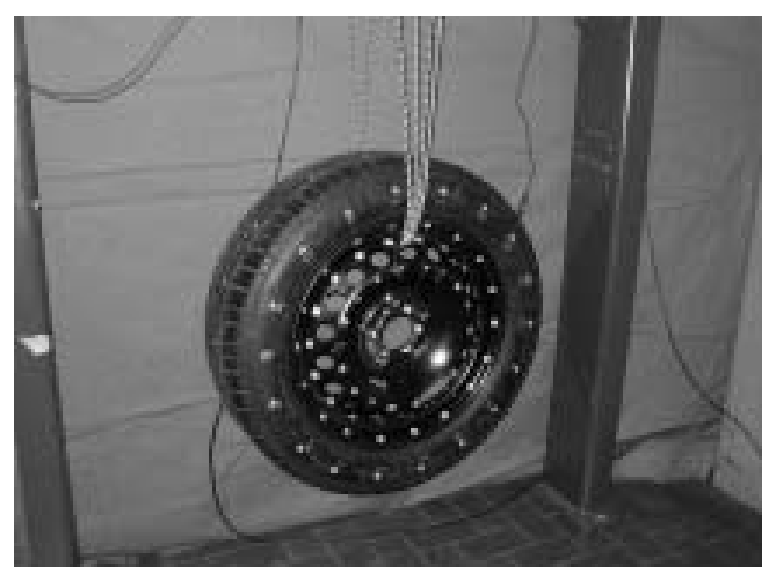

Fig. 1. Saab wheel with free-free condition.

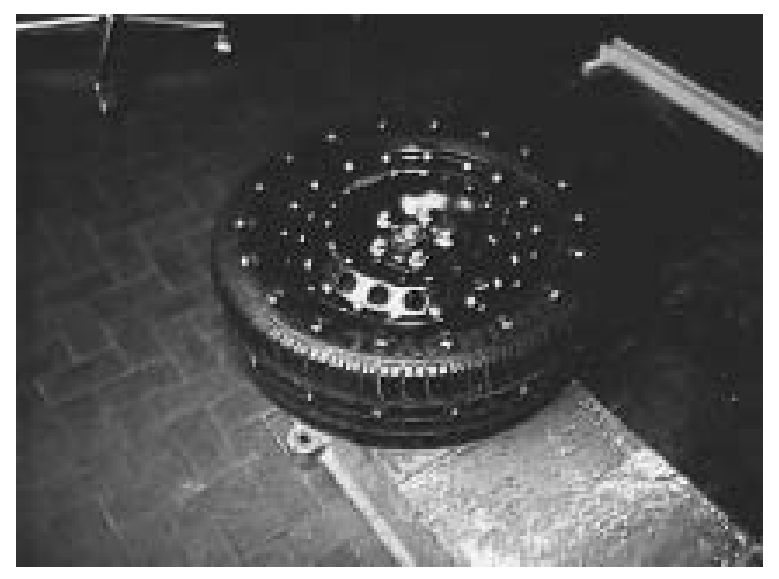

Fig. 2. Saab wheel clamped to the test hub.

technique has been employed because of the difficulty to excite the nodes on the tire surface; the impulse force has been provided in radial direction to the node 77 of the rim and in axial direction to the node 22 [1] (see Fig. 3).

The impulse force (F) has been provided by a Bruel \& Kjaer 8200 hammer (charge sensitivity 4 pC/N). The acceleration (a) has been measured by a Bruel \& Kjaer 4393 accelerometer (charge sensitivity 0.315 Pc/ms -2 respectively) in both radial and axial directions.

The force and acceleration signals have been processed by a spectrum analyser (DIFA APB200 and LMS International Modal Analysis Software) for the evaluation of the $\mathrm{a} / \mathrm{F}$ transfer functions in all measure points. 


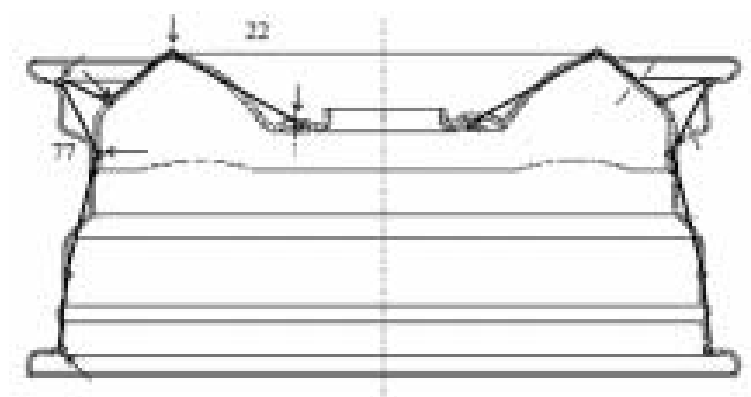

Fig. 3. Experimental model.

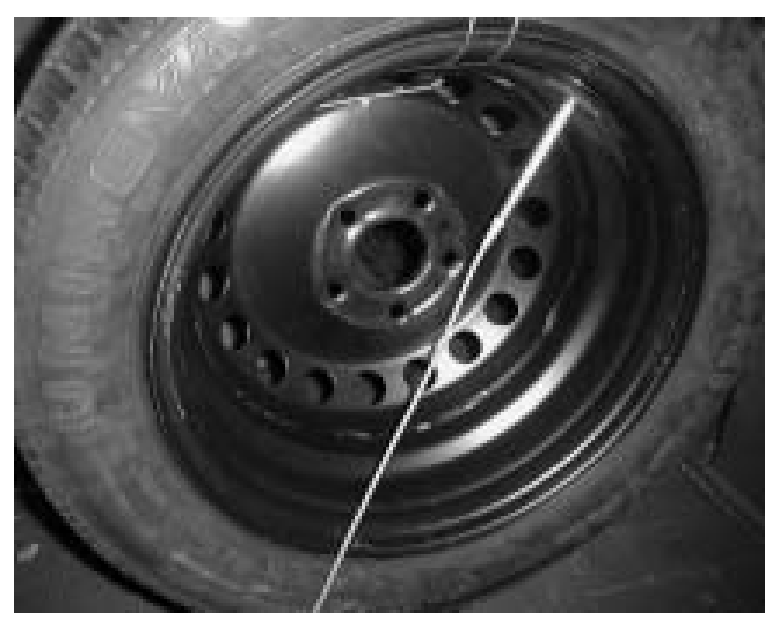

Fig. 4. Acoustic analysis of the Saab wheel.

By employing the LMS Software, the modal parameters of the wheels (i.e. natural frequencies, mode shapes) have been also obtained.

The analysis has been performed in a frequency range $0-1600 \mathrm{~Hz}$.

The acoustic analysis of the tire cavity has also been carried on. To do that, another SAAB rim with the Uniroyal tire (with free-free condition) has been considered: a microphone has been introduced into the cavity $(p=0)$ in order to measure the acoustic Frequency Response Function (see Fig. 4). A random structural excitation in axial direction has been provided by an electrodynamic shaker Bruel \& Kjaer 4810 connected through a stinger to a point of the disc. The excitation spectrum was a band-limited noise $(0-1200 \mathrm{~Hz})$. Both acceleration (of an accelerometer placed on the tread) and acoustic signals have been measured.

\section{Theoretical and numerical models}

The analytical model of the tire cavity utilised to calculate both natural frequencies and mode shapes is a tubular cavity with rigid walls. The close form solution is [10]:

$$
f_{i j k}=\frac{c}{2 \pi} \sqrt{\left(\frac{i \pi}{L}\right)^{2}+\left(\frac{\lambda_{j, k}}{R_{1}}\right)^{2}}
$$

where $i, j, k$ are integers, $c$ is the sound velocity, $L$ is the cavity thickness and $R_{1}$ is the internal radius of the section; $\lambda_{j k}$ are the eigenvalues of the system, functions of $i, j, k$ and of the ratio $R_{2} / R_{1}$, where $R_{2}$ is the external radius of the section; $i$ indicates the number of axial nodes, $j$ the number of nodal diameters and $k$ the number of nodal circumferences. 


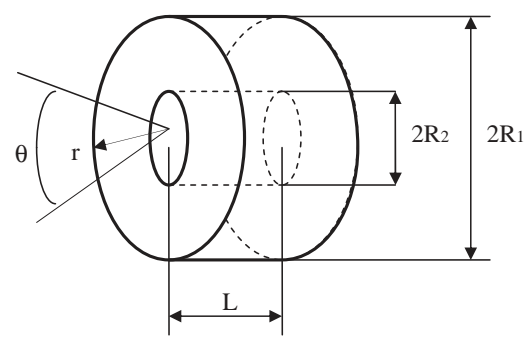

Fig. 5. Theoretical model of the Uniroyal tire cavity.

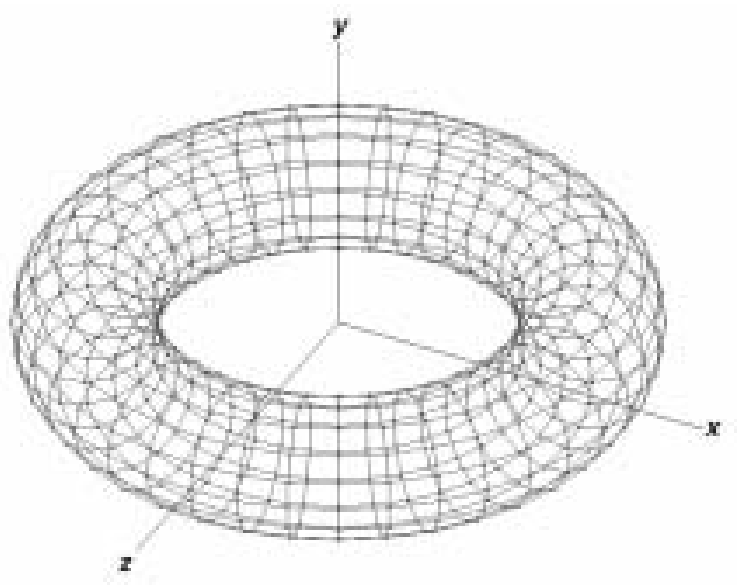

Fig. 6. BEM model of the Uniroyal tire cavity.

An equivalent geometrical model of the Uniroyal tire has been considered (see Fig. 5), where $R_{1}=287.4 \mathrm{~mm}$, $R_{2}=190.4 \mathrm{~mm}$ and $L=195 \mathrm{~mm}$. Both acoustic frequencies and corresponding mode shapes have been calculated in the frequency range $0-1200 \mathrm{~Hz}$.

The numerical calculations of both natural frequencies and mode shapes of the tire cavity have been performed by means of the Vnoise BEM code (PC release). The cavity BEM model has been built-up by 640 linear surface elements with 4 nodes and 10 calculation points for each wave length at the maximum frequency, in the range $100-800 \mathrm{~Hz}$ (step $1 \mathrm{~Hz}$ ) (see Fig. 6).

\section{Results}

The results of the research activity performed in this paper are shown in Tables 1 to 7 .

Each Table shows in increasing order both frequencies and mode shapes corresponding to each condition of the Saab wheel.

In particular Tables 1, 2, 3, 4, 5 show results obtained in the laboratory tests and Tables 6, 7 show results obtained on the Saab vehicle [3].

Table 1 shows the frequencies and the corresponding mode shapes of the Uniroyal tire (pressure $p=2.5 \mathrm{~atm}$ ); there are two set of measured data involving similar frequency values: the first column shows the frequency values obtained by processing the response signals (acceleration/force) coming from the measure points over the tire (64 nodes), while the second column shows the corresponding values obtained by processing all the nodes of the experimental model (rim + tire) (168 nodes).

The obtained results are quite similar, except of the 6th tire mode that has not been emphasised with the whole model (168 nodes). 
Table 4

Natural frequencies and mode shapes of the Saab wheel (Saab rim, Uniroyal tire $p=2.5 \mathrm{~atm}$ ) with free-free condition (bold faced, free-free rim modes; *, repeated eigenvalues; + , modes coming from the tire; $(w)$, wheel modes)

\begin{tabular}{|c|c|}
\hline Frequency $[\mathrm{Hz}]$ & Mode shape \\
\hline 70 & axial rigid motion,+ 1 st tire mode \\
\hline 110 & 2 lobes, 1 st mode,+ 2 nd tire mode \\
\hline 113 & 2 lobes, 1 st mode $+*, 2$ nd tire mode $*$ \\
\hline 136 & rim flanges bending,+ 3 rd tire mode \\
\hline 193 & 4th tire mode* \\
\hline 206 & 2 lobes, 1st mode \\
\hline 236 & rim flanges bending,+ 010 cavity mode \\
\hline 409 & 3 lobes, 1 st mode,+ 5 th tire mode \\
\hline 436 & 6th tire mode \\
\hline 450 & 020 cavity mode \\
\hline 463 & 7 th tire mode \\
\hline 529 & 3 lobes, 1st mode \\
\hline 686 & 3 lobes, 2 nd mode, 030 cavity mode, 8 th tire mode \\
\hline 756 & 9th tire mode \\
\hline 810 & 10th tire mode \\
\hline 915 & 4 lobes, 1 st mode, 040 cavity mode \\
\hline 923 & 4 lobes, 1 st mode* \\
\hline 1038 & 4 lobes, 2nd mode \\
\hline 1078 & pumping, 111 cavity mode \\
\hline 1125 & 11 th tire mode, 130 cavity mode \\
\hline 1160 & disc oscillation (w), 050 cavity mode \\
\hline
\end{tabular}

Table 5

Natural frequencies and mode shapes of the Saab wheel (Saab rim, Uniroyal tire $p=2.5 \mathrm{~atm}$ ) with clamped condition (tightening torque $C=13 \mathrm{kgm}$ ) (bold faced, free-free rim modes; *, repeated eigenvalues; +, modes coming from the tire; $(\mathrm{w})$, wheel modes; (c) modes coming from the clamping devise)

\begin{tabular}{|c|c|}
\hline Frequency $[\mathrm{Hz}]$ & Mode shape \\
\hline 54 & rim-clamp, 1 st mode $(c)$ \\
\hline 72 & rim-clamp, 2 nd mode $(c), 1$ st tire mode \\
\hline 83 & rim-clamp, $3 r d$ mode $(c)$ \\
\hline 87 & rim-clamp, 3rd mode * (c) \\
\hline 111 & 2 lobes, 1 st mode,+ 2 nd tire mode \\
\hline 131 & rigid rotation,+ 3 rd tire mode \\
\hline 182 & rim-clamp, 4 th mode $(c)$ \\
\hline 192 & 4th tire mode \\
\hline 208 & 2 lobes, 1 st mode \\
\hline 237 & rim flanges bending, pumping $(c), 010$ cavity mode \\
\hline 410 & 3 lobes, 1 st mode,+ 5 th tire mode \\
\hline 438 & 6th tire mode \\
\hline 454 & 020 cavity mode \\
\hline 529 & 3 lobes, 1st mode \\
\hline 682 & rim flanges bending, pumping $(c), 030$ cavity mode, 8 th tire mode \\
\hline 760 & 9th tire mode \\
\hline 914 & rim flanges bending (w), 040 cavity mode \\
\hline 974 & 4 lobes, 1st mode \\
\hline 1128 & disc oscillation (w), 11 th tire mode, 130 cavity mode \\
\hline
\end{tabular}

In Table 2 the results of the acoustic analysis are reported, that is the natural frequencies and the corresponding mode shapes of the tire cavity. In particular the second and third column refer respectively to the analytical and numerical (BEM model) results; the fourth column shows the experimental results coming from the microphone measurements.

Table 2 shows a good agreement between analytical, numerical (BEM) and experimental results. It may be pointed out that BEM solution emphasises only radial modes (that is modes with nodal diameters) and acoustic tests emphasises only modes with predominant components in radial direction (see Fig. 4). 
Table 6

Natural frequencies and mode shapes of the Saab wheel (Saab rim, Uniroyal tire) clamped on the vehicle hub (hanging condition) (bold faced, free-free rim modes; + , modes coming from the tire; (c) modes coming from the clamping devise)

\begin{tabular}{|c|c|c|}
\hline \multicolumn{2}{|c|}{ Natural frequency $[\mathrm{Hz}]$} & \multirow[t]{2}{*}{ Mode shape } \\
\hline Impulse excitation & Random excitation & \\
\hline 54 & 54 & rim-clamp, 1 st mode $(c)$ \\
\hline 84 & 84 & rim-clamp, 2 nd mode $(c), 1$ st tire mode \\
\hline 125 & 125 & rim-clamp, $3 r d$ mode $(c)$ \\
\hline 155 & 140 & rim-clamp, 4 th mode (c) \\
\hline- & 170 & 2 lobes, 1 st mode + \\
\hline 185 & 178 & rigid rotation + \\
\hline 212 & 212 & 2 lobes, 1st mode \\
\hline 250 & 250 & rim flanges bending, pumping (c) \\
\hline 270 & 270 & rim flanges bending, pumping (c) \\
\hline- & 300 & axial rigid motion + \\
\hline 410 & 450 & 3 lobes, 1 st mode,+ 020 cavity mode \\
\hline- & 671 & rim flanges bending, pumping $(c), 030$ cavity mode, 8 th tire mode \\
\hline
\end{tabular}

Table 7

Natural frequencies and mode shapes of the Saab wheel (Saab rim, Uniroyal tire) clamped on the vehicle hub (wheel laying on the ground) (bold faced, free-free rim modes; + , modes coming from the tire; (c) modes coming from the clamping devise)

\begin{tabular}{cc}
\hline $\begin{array}{c}\text { Natural frequency }[\mathrm{Hz}] \\
\text { Random excitation }\end{array}$ & Mode shape \\
\hline 55 & rim-clamp, 1st mode $(c)$ \\
- & rim-clamp, 2 nd mode $(c), 1$ st tire mode \\
125 & rim-clamp, $3 r d$ mode $(c)$ \\
150 & rim-clamp, 4 th mode $(c)$ \\
- & 2 lobes, 1st mode + \\
178 & rigid rotation + \\
$\mathbf{2 0 6}$ & $\mathbf{2}$ lobes, 1st mode \\
250 & rim flanges bending, pumping $(c)$ \\
270 & rim flanges bending, pumping $(\mathrm{c})$ \\
315 & axial rigid motion + \\
450 & 3 lobes, 1 st mode,+ 020 cavity mode \\
671 & rim flanges bending, pumping $(c), 030$ cavity mode, 8 th tire mode \\
\hline
\end{tabular}

Table 3 shows (in bold-faced) the frequencies and the corresponding mode shapes of the free-free Saab rim (already measured in a previous research [1-3]).

Radial mode shapes have been classified for increasing both the number of lobes (one lobe, two lobes, ...) and the order of bending modes (first mode, second mode, ...); axial mode shapes are called "pumping" and "oscillation", involving only an axial motion of the disc.

The mode shapes denoted with asterisk $\left(^{*}\right)$ refer to similar modes, corresponding to repeated eigenvalues, rotated with respect to the rim axis of $40-45^{\circ}$ for two lobes and of $20-25^{\circ}$ for four lobes modes respectively. This phenomenon can be attributed to the structural asymmetry due to four weld beads connecting disc and rim together.

With reference to two lobes similar modes, it can be observed that the nodes of the first mode correspond to the centre of the weld beads and the nodes of the second one correspond to the centre of the non welded zones.

Table 4 shows the natural frequencies and the corresponding mode shapes of the free-free Saab wheel (rim with tire) $(p=2.5 \mathrm{~atm})$. It may be pointed out that the frequencies corresponding to the free-free rim (without tire) modes have been identified in bold-faced in all Tables (Tables 3 to 7).

In the same Table it is possible to identify the rim frequencies due to the presence of the tire (modes denoted with the symbol "+"). In particular, the first mode of the rim with tire corresponds to a rigid motion of the rim (coupled to a tire motion), radial mode shapes (one lobe, two lobes, ... ) correspond to a deformation of both rim and tire and the "rim flanges bending" modes can be considered as local modes.

Table 4 shows also both structural modes of the tire (1st tire mode, 2nd tire mode, ...) (see Table 1, second column) and acoustic modes of the cavity ( 010 cavity mode, 020 cavity mode, ...) (see Table 2 , fourth column). 


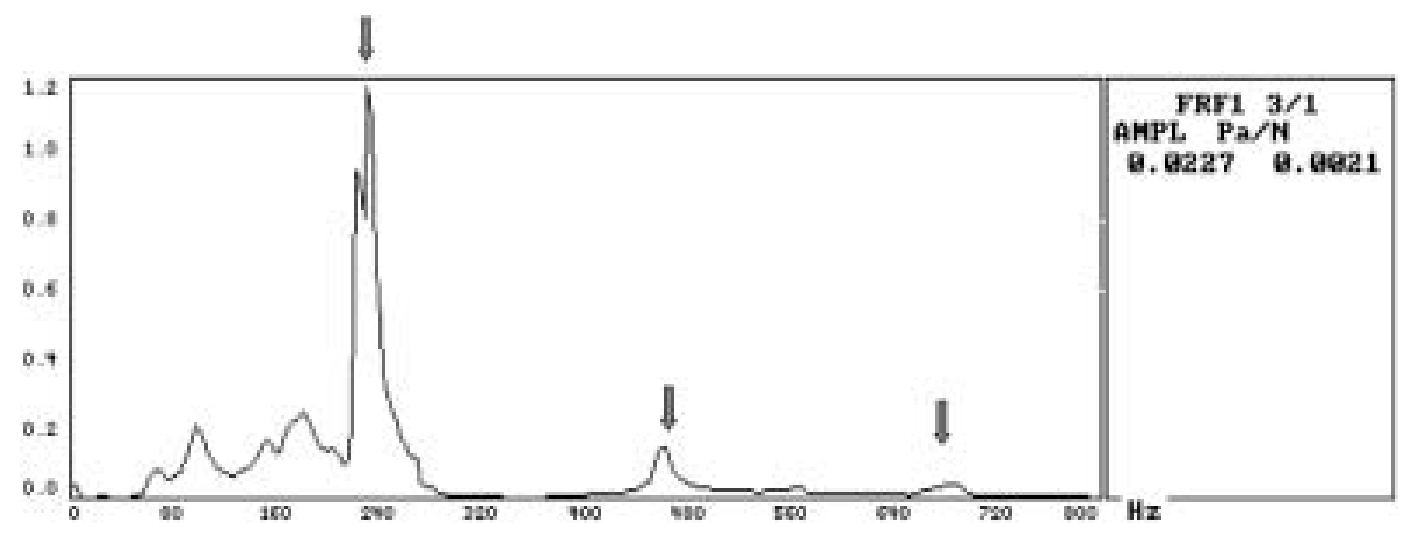

Fig. 7. p/F Frequency Response Function of the Uniroyal tire cavity.

Finally it can also be noted an axial mode shape of the whole wheel (rim with tire) (bold-faced and italic, denoted with the symbol "w") identified as "disc oscillation".

Table 5 shows the natural frequencies and the corresponding mode shapes of the Saab wheel (rim with tire) clamped to the test hub (tightening torque $C=13 \mathrm{Kgm}, p=2.5 \mathrm{~atm}$ ).

In Table 5 it can be observed both modes corresponding to the free-free Saab wheel (Table 4) and new modes of the wheel due to the clamped condition (italic, denoted with the symbol "c"). The mode shapes denoted as "rim-clamp modes" (first rim-clamp mode, second rim-clamp mode, ... ) involve a motion not only of the rim but also of the clamp (as detectable by an accelerometer signal placed on the hub). Otherwise the mode shapes denoted as "pumping" involve only a rim motion. It can be pointed out that probably only the above quoted "rim-clamp modes" can be influenced by the clamp stiffness.

In Table 6 are reported the natural frequencies measured on the hanging wheel. In first and in second column respectively the results of both impulse and random excitation tests are shown.

Table 7 concerns with the natural frequencies of the wheel laying on the ground. This test was performed with random excitation only.

In Tables 6 and 7 are also classified the mode shapes corresponding to the above quoted natural frequencies. These mode shapes are identified only on the basis of the experience gained in the laboratory tests (both in [1] and [2] and in the present work). As a matter of fact, the poor number (due to the vehicle body) of measure points utilised in the experimental model on the vehicle [3] didn't allow to process the measured data by means of LMS Software in order to obtain adequate mode shapes.

It can be pointed out that all the natural frequencies of the wheel clamped to the vehicle hub in hanging condition (Table 6) are a little higher than the corresponding obtained in the laboratory tests.

This behaviour, pointed out with both impulse and random excitation, is common to all mode shapes above classified (modes of the free-free rim, modes of the rim due to the clamped condition and modes of the rim due to the tire).

Table 6 shows that the natural frequencies obtained by means of both random and impulse tests are practically equal, even if the impulse analysis is not able to point out three modes of the system.

As a matter of fact, the impulse excitation provides a lower amount of energy with respect to the random one and therefore this technique is not so able to excite the wheel clamped to the vehicle: in general it is very difficult or, in some cases, quite impossible to obtain the natural frequencies from the analysis of the measured data.

It can be pointed out that the natural frequencies obtained by means of the random excitation in both cases of hanging (Table 6) and laying (Table 7) wheel are a little different.

Figure 6 shows the Frequency Response Function (p/F) of the Uniroyal tire cavity in the frequency range $0-800 \mathrm{~Hz}$.

As an example, Figs 9, 10 and 11 show some mode shapes of the Saab wheel obtained by processing the measured data.

In particular, Fig. 9 shows the second mode shape of the Uniroyal tire (2 lobes, 1 st mode + , 2nd tire mode) $(111 \mathrm{~Hz})$. 


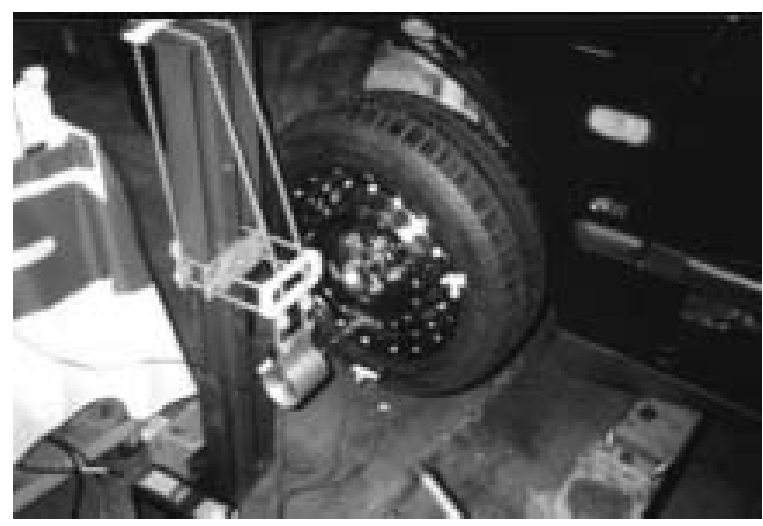

Fig. 8. Saab wheel clamped to the vehicle hub.

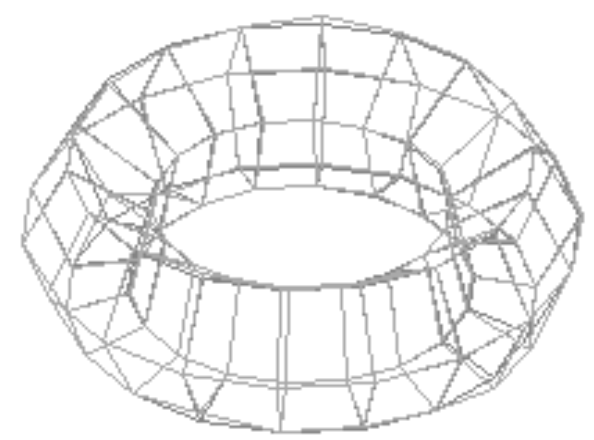

Fig. 9. Second mode shape of the Uniroyal tire (2 lobes, 1 mode,+ 2 tire mode) $(111 \mathrm{~Hz})$.

Figure 10 shows the two lobes mode shape of the clamped Saab wheel (114 measure points) ( 2 lobes, 1 st mode) $(208 \mathrm{~Hz})$.

Figure 11 shows the three lobes mode shape of the clamped Saab wheel (168 measure points) ( 3 lobes, 1 st mode) $(529 \mathrm{~Hz})$.

\section{Conclusions}

The present research activity concerning the dynamic and acoustic characterisation of automotive wheels allows one to draw the following general conclusions.

Experimental modal analysis technique provides a most satisfactory and repeatable evaluation of the dynamic parameters (natural frequencies and mode shapes) of automotive wheels.

The experimental results obtained from modal analysis tests performed in the laboratory provides a classification of the mode shapes: free-free rim modes, rim-tire modes, rim-clamp modes, tire modes and, finally, rim-tire-clamp modes. The employed test procedure allows us to characterise all natural frequencies and corresponding mode shapes.

It may be observed that the dynamic behaviour of the rim is substantially modified by the tire, both as regards frequency values and as regards amplitude levels (frequency response functions have been omitted in this work for sake of brevity); only axial mode shapes amplitudes are not influenced by the presence of the tire.

Also the clamping conditions substantially modify the dynamic behaviour of the rim involving a variation of both free-free rim frequencies and frequencies of the rim-clamp system. 

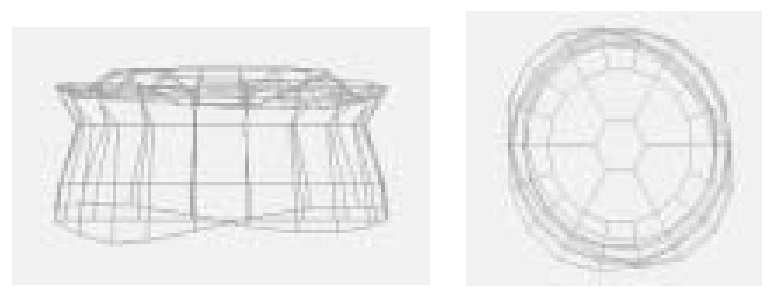

Fig. 10. Two lobes mode shape of the clamped Saab wheel (114 measure points) (2 lobes, 1st mode) (208 Hz).

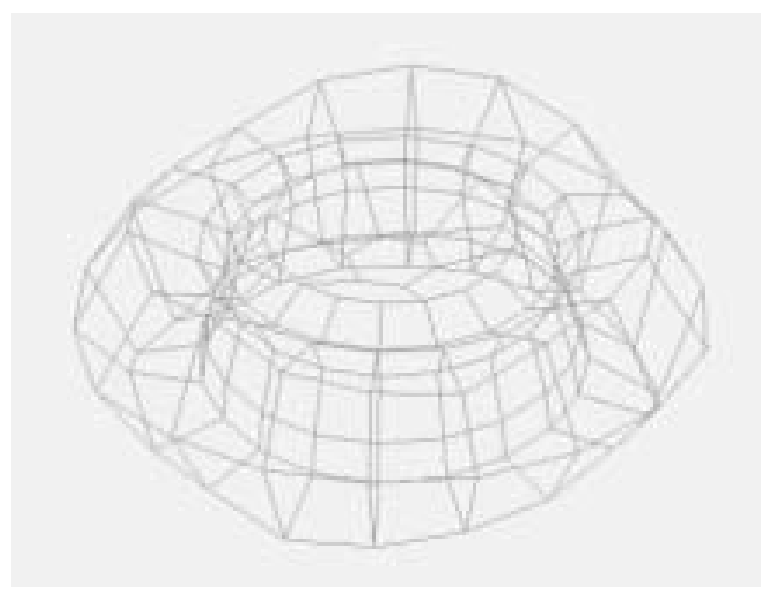

Fig. 11. Three lobes mode shape of the clamped Saab wheel (168 measure points) (3 lobes, 1st mode) $(529 \mathrm{~Hz})$.

The excitation procedure of the wheel clamped to the vehicle in general presents some difficulties and has to be limited to measure points reachable by the shaker. In this situation it is quite impossible to characterise all mode shapes corresponding to the recorded natural frequencies.

However, the research activity carried on step by step allows us to explain the signals measured on the vehicle on the basis of the laboratory tests, in order to certainly evaluate the dynamic behaviour of the wheel, that is the natural frequencies and the corresponding mode shapes.

It may be pointed out that all the natural frequencies of the wheel clamped to the vehicle in hanging condition are a little higher than the corresponding obtained in the laboratory tests (see Table 6): this is probably due to the stiffness of the vehicle hub.

It may be also observed that the dynamic behaviour of the wheel clamped to the vehicle is substantially similar in both hanging and laying condition (see Tables 6 and 7), even if some differences may be highlighted in the recorded signals (that have been omitted for sake of brevity).

Moreover, vibrations of a wheel may be influenced both by structural and acoustic modes. As a matter of fact, isochronism conditions between acoustic and/or structural resonances (as an example, Table 6 shows the isochronism condition between the 3 lobes, 1 st mode + and the 020 cavity mode) can contribute to increase both vibrations and produced noise level.

To sum up, the knowledge of both dynamic and acoustic parameters already in design phase can allow to avoid some vibrations and noise problems arising during the vehicle running.

\section{Acknowledgements}

This research activity was made possible by the partial contribution of FERGAT S.p.A.

The experimental tests on the vehicle were made possible by the collaboration of SIDAUTO S.p.A. Many thanks to Mrs. Giulia Pagani Mamiani della Rovere. 
Many thanks also to Mr. Di Francescantonio who kindly provided the software Vnoise to performe a Tesi di Laurea at Politecnico di Torino.

\section{References}

[1] G. Curti and F. Curà, Analisi sperimentale del comportamento dinamico di ruote per autoveicoli, Atti del XXVII Convegno Nazionale AIAS, Perugia I (8-12 settembre 1998), 551-560.

[2] G. Curti and F. Curà, Experimental Analysis of Automotive Wheels, Paper n 99A4118, ATA Int. Conf., Florence, november 1999.

[3] G. Curti and F. Curà, Experimental Dynamic Characterisation of a Wheel Clamped to a Vehicle, Paper ip 01A1026, ATA Int. Conf., Florence, november 2001.

[4] R.W. Scavuzzo, L.T. Charek, P.M. Sandy and G.D. Shteinhauz, Influence of Wheel Resonance on Tire Acoustic Cavity Noise.

[5] L.E. Kung and W. Soedel, Free vibration of a pneumatic tire wheel using a ring on an elastic foundation and a finite element model, Journal of Sound and Vibration 107(2) (1986), 181-194.

[6] T.L. Richards, Finite element analysis of a structural-acoustic coupling in tires, Journal of Sound and Vibration 149(2) (1991), $235-243$.

[7] LMS CADA X, Manuale Tecnico.

[8] D.J. Ewins, Modal Testing: Theory and Practice, Yarmouth (GB), Research Studies Press, 1989.

[9] S. Markus, The Mechanics of Vibrations of Cylindrical Shells, Amsterdam, Elsevier Science Publisher, 1988.

[10] E. Zagatti, R. Zennaro and P. Pasqualetti, L'assetto dell'autoveicolo - Sospensioni - Pneumatici - Sterzatura - Comportamento dinamico, Torino, Levrotto \& Bella, 1994.

[11] K.G. Mc Connel, Vibration Testing: Theory and Practice, New York, Wiley International Science, 1995.

[12] R.D. Blevins, ormulas for natural frequency and mode shape, Van Nostrand, New York, 1979.

[13] A. Bassanino, Sperimentazione numerica nell'analisi vibrazionale di una ruota per autoveicolo, Tesi di Laurea in Ingegneria Meccanica, Politecnico di Torino, Dicembre 2001. 

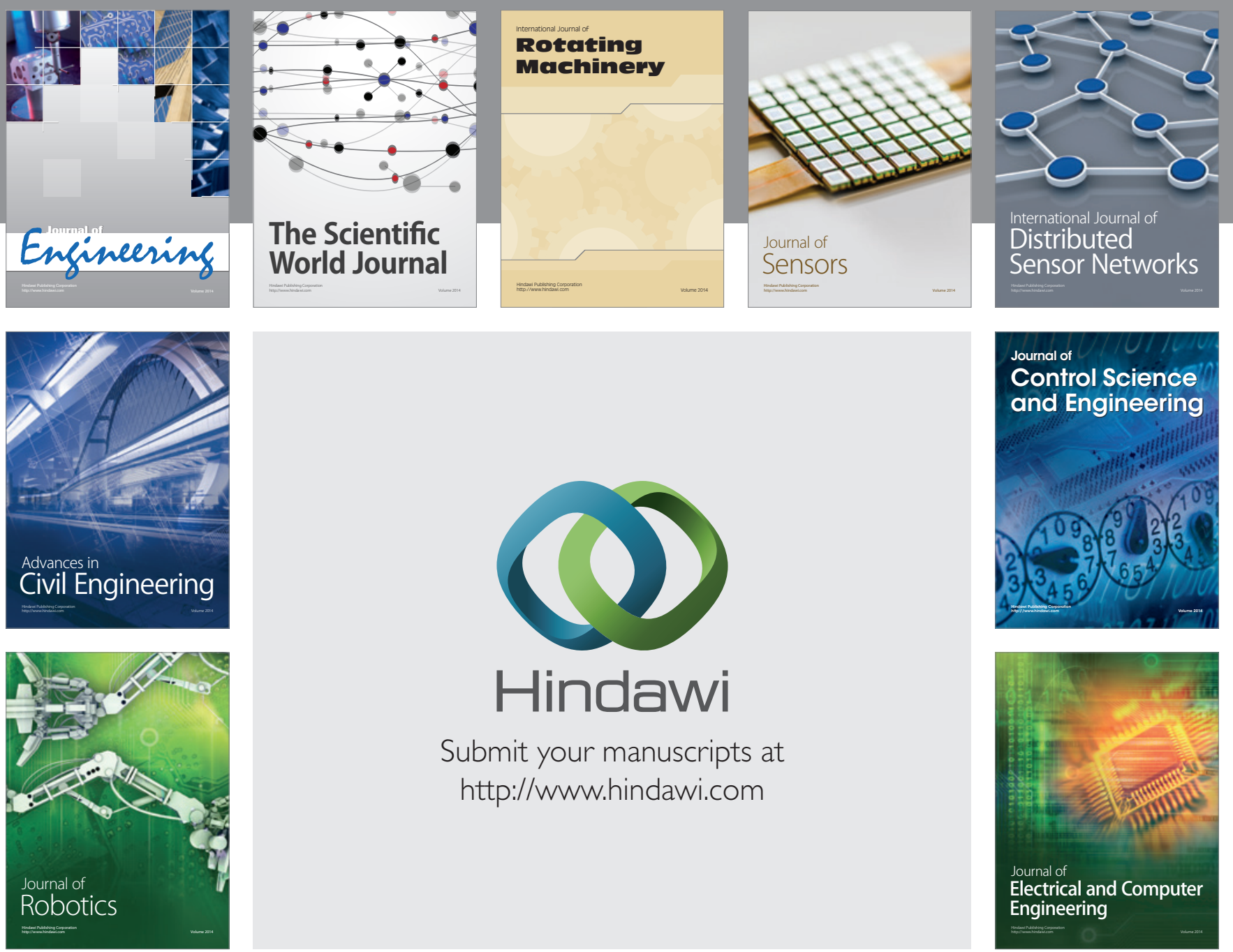

Submit your manuscripts at

http://www.hindawi.com
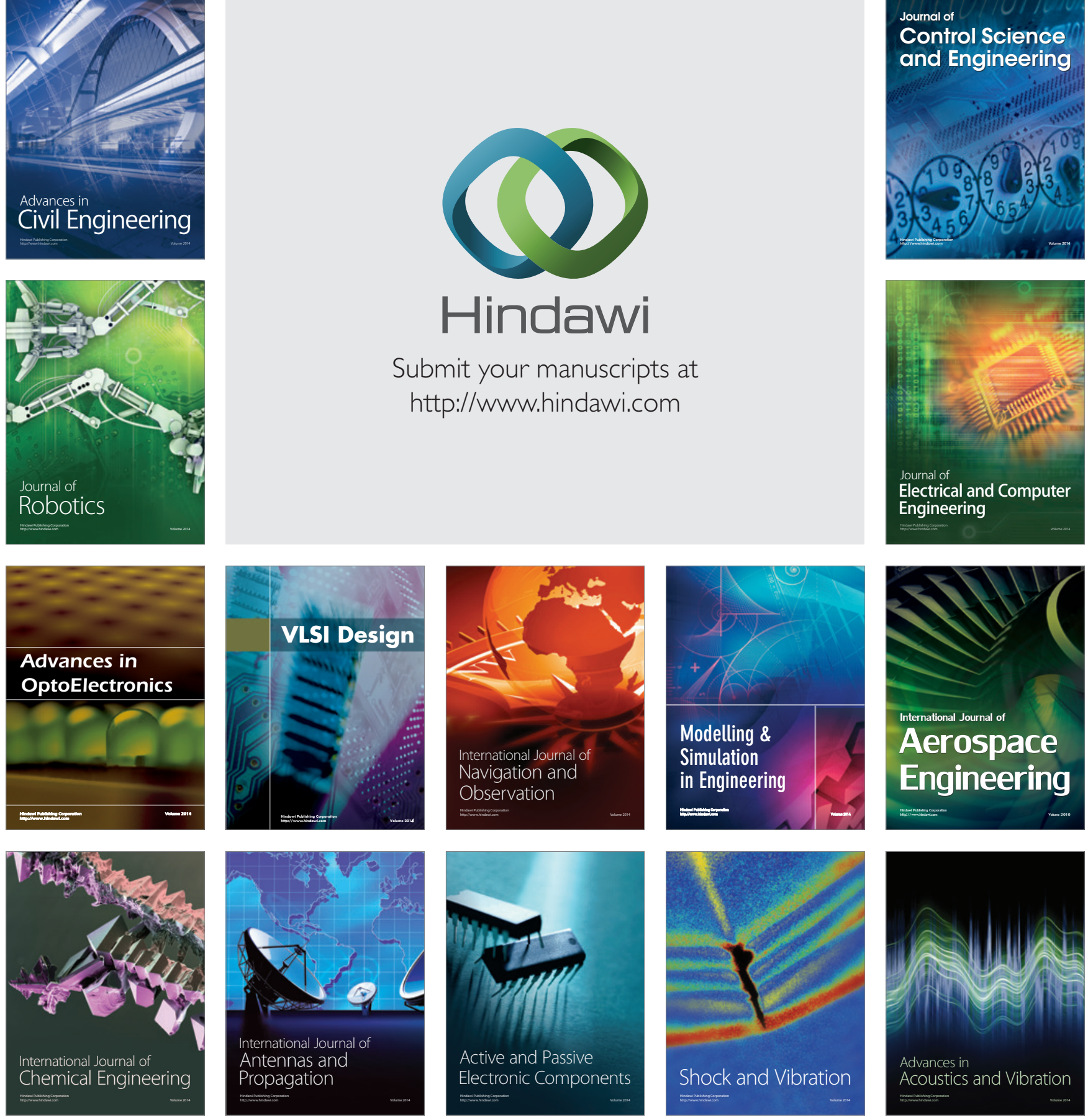